1 Faculty of Public Health Cite this as: BMJ 2021;375:n3095 http://dx.doi.org/10.1136/bmj.n3095 Published: 16 December 2021

\title{
Omicron: a failure to act with a global focus will continue the proliferation of new variants of covid-19
}

\section{Maggie Rae president}

The emergence of the omicron variant of covid-19 serves as a stark reminder that while we have made progress in tackling covid-19 over the past two years, there is still much work to be done. We are anticipating that omicron becomes the dominant variant across the UK in a matter of weeks. When we step back from a UK focused perspective and look at the pandemic from a global perspective, the dominance of the omicron variant becomes even plainer. While we must continue to implement public health measures to protect our domestic population, if we fail to act with a global focus we will continue to see the proliferation of new variants of the virus.

Inequity is the common denominator in many of the challenges faced by public health professionals, and this rings true for our attempts to tackle the covid-19 pandemic. While $80 \%$ of the UK population is fully vaccinated, this figure stands at only $6.6 \%$ for the African continent. ${ }^{1}$ While we still seek a definitive answer as to where the omicron variant of covid-19 originated, what is certain is that unless populations across the world are protected from the threat of the virus, we will continue to see new variants emerge and spread internationally.

Our most important call to governments then, is to support global vaccination efforts through lifting import restrictions and intellectual property protections, while also resourcing and supporting vaccine delivery programmes in low-income countries. Covid-19 is not a short term problem; our aim as a global community must be to learn to live alongside the virus, and to achieve this goal requires coordinated international action based on principles of equity.

This being understood, we must also take every action we can to control the domestic spread of the virus. Vaccination is undoubtedly our strongest tool in tackling this pandemic, with early data suggesting that a third booster dose prevents symptoms from omicron in $75 \%$ of people. ${ }^{2}$ However, just as in the international context, we see inequalities play out in our efforts to drive forward vaccination within our domestic population. We know that people from ethnic minority groups are less likely to be vaccinated than those identifying as White British, and data from the Office for National Statistics (ONS) show us that those in the most deprived areas are over twice as likely to be unvaccinated as those in the least deprived. ${ }^{3}$ To protect our domestic population from covid-19, the government must support vaccine equity by removing barriers to access for these groups, including through better community engagement, clearer messaging, and financial support where necessary.
As well as re-doubling efforts to support vaccine uptake across the UK population, the government must ensure it is delivering clear and accurate public health messaging. Throughout the course of the pandemic senior government officials have been forced to resign their posts for contradicting covid-19 restrictions; an unhelpful distraction when public adherence to covid-19 guidance is so critical. Without confidence in public health messaging, we effectively lose the ability to implement the evidence based measures necessary to control the spread of the virus, protect the NHS, and limit damage to our economy.

In terms of the measures themselves, the Faculty of Public Health and our partners have long-called for the mandatory wearing of facemasks in settings where the virus can easily spread. Good hand hygiene, including the use of hand gels, remains equally as important and the government must do more to remind the public of this. People must also be encouraged to be responsible when meeting friends and family over the festive period; keeping gatherings small if possible and remembering to regularly air out rooms if meeting indoors. While rules on isolation have been relaxed for fully vaccinated contacts of those who have contracted coronavirus, with infection rates rocketing we must offer better support to those who have tested positive and are required to isolate.

Public health professionals will work over the Christmas period, alongside health and care staff, to protect the health of the populations they serve. The emergence of the omicron variant of covid-19 throws into sharp relief the actions necessary to support these efforts. Governments across the world must come together to ensure international vaccine equity and protect the health of our global community. We also need to address domestic inequalities in vaccine uptake, and the government must re-double efforts to ensure that those not already vaccinated get their jabs. Damaged confidence in public health messaging delivered by the government must be repaired, and we must ensure that people follow current guidance if we are to control the spread of the virus and prevent further lockdowns.

\footnotetext{
Competing interests: none declared

Provenance and peer review: commissioned, not peer reviewed

Our World in Data. Coronavirus (COVID-19) Vaccinations. https://ourworldindata.org/covid-vaccinations

2 BBC News. Omicron: Three vaccine doses key for protection against variant https://www.bbc.co.uk/news/health-59615005

Office for National Statistics. COVID-19 vaccination rates and odds ratios by socio-demographic group https://www.ons.gov.uk/peoplepopulationandcommunity/healthandsocialcare/healthinequalities/datasets/covid19vaccinationratesandoddsratiosbysociodemographicgroup
} 